\title{
Can Cats Cause Schizophrenia? An Insight into the Role of Bartonella Henselae in Neuropsychiatric Disorders
}

\author{
Divya $\mathbf{R}^{\mathbf{1}}$ and Rajajeyakumar $\mathbf{M}^{2 *}$ \\ ${ }^{1}$ Assistant Professor, Department of Physiology, Karpagam Faculty of Medical Sciences and Research, Coimbatore, India \\ ${ }^{2}$ Assistant Professor, Department of Physiology, Trichy SRM Medical College Hospital and Research Centre, Trichy, India \\ *Corresponding Author: Rajajeyakumar M, Assistant Professor, Department of Physiology, SRM Medical College Hospital and Research \\ Centre, Trichy, MGR Medical University, Chennai, Tamil Nadu, India.
}

Received: April 25, 2019; Published: June 20, 2019

DOI: $10.31080 /$ ASPS.2019.03.0322

\section{Cat Scratch Disease(CSD)}

Bartonella henselae is a fastidious gram-negative rod that is the etiologic agent of Cat Scratch Disease(CSD) and is allied with bacillary angiomatosis in HIV-infected individuals. A total of 30 different species of Bartonella and 13 of those have been found to infect human beings [1]. Different Bartonella species causes wide-ranging and multifaceted diseases in humans [2]. The primary carriers of Bartonella henselae are Cats. Stray cats as well as healthy pets. The flea vector Ctenocephalides felis transmits Bartonella between cats. The mode of transmission to humans is through a scratch or bite from a cat or a kitten. Cat scratch disease is prevalent in all age groups, but more common in children less than 10 years of age [3].

Neurobartonellosis

In Humans, the bacterium infects pericytes, macrophages, erythrocytes, and endothelial cells, resulting in vascular pathology. Throughout the world, research data demonstrates a broad spectrum of neurological abnormalities caused by Bartonella spp referred to as neurobartonellosis. It is found to have equal prevalence in immunocompetent and immunocompromised patients [4]. Bartonella species were isolated from chronically ill patients, suffering from various diseases such as epilepsy, rheumatoid arthritis and migrane with idiopathic etiology [1].

Pediatric Acute-onset Neuropsychiatric Syndrome (PANS): A case Study

Due to a sudden onset psychotic behaviour, an adolescent patient was diagnosed and treated for schizophrenia over a period of 18 months; resulting in failure of psychosis and autoimmune disorders treatment. The lesions on the patient's skin were identified and led to the diagnosis of serum positive Bartonella infection. The adolescent was then treated with Long term antimicrobial chemotherapy that helped in full recovery from all the Neuropsychiatric Symptoms. This study advocates the contribution of Bartonella infection towards the development and progression of neuropsychiatric disorders such as schizophrenia [1].

\section{Conclusion}

This advancement has led to a different aspect of approach towards the infectious etiology of neuropsychiatric illnesses such as Schizophrenia, Alzheimer's disease. Future investigations are essential to understand the potential role of viral and bacterial infections in medically complex neuropsychiatric disorders with idiopathic etiology.

\section{Bibliography}

1. Breitschwerdt EB., et al. "Bartonella henselae Bloodstream Infection in a Boy with Pediatric Acute-Onset Neuropsychiatric Syndrome". Journal of Central Nervous System Disease (2019).

2. Vayssier-Taussat M., et al. "Identification of Novel Zoonotic Activity of Bartonella spp., France". Emerging Infectious Diseases 22.3 (2016): 457-462.

3. Mogollon P., et al. "Bartonella: emerging pathogen or emerging awareness?". International Journal of Infectious Diseases 13.1 (2009): 3-8.

4. Balakrishnan N., et al. "Vasculitis, cerebral infarction and persistent Bartonella henselae infection in a child". Parasit Vectors 9.1 (2016): 254.

Volume 3 Issue 7 July 2019

(C) All rights are reserved by Divya $\mathrm{R}$ and

Rajajeyakumar M. 